\title{
Genetic and pathophysiological substantiation of polyphenolic grape processing products' application in the treatment of metabolic syndrome in the population of the Republic of Crimea
}

\author{
Iuliana Shramko*, Elizaveta Ageeva, Konstantin Maliy, Irina Repinskaya, and Anna \\ Gurtovaya
}

S. I. Georgievsky Medical Academy of the Federal State Autonomous Educational Institution of Higher Education " V. I. Vernadsky Crimean Federal University», 295000, Simferopol, Russia

\begin{abstract}
Adipose tissue in abdominal obesity produces various cytokines, the most important of which is adiponectin (AN). Polymorphism of the AN receptor genes is associated with the risk of metabolic syndrome (MS) and type 2 diabetes mellitus (DM2) development. The aim of the study was to investigate the association of AN genes receptors' polymorphism with the development of MS and DM2 in the Republic of Crimea as well as the possibility of named pathology correction with polyphenolic functional foods. The study included 100 patients with confirmed diagnosis of DM2 and MS. Genomic DNA was isolated from the whole blood of patients. Gene polymorphism amplification was performed by PCR real-time. The most common genotype of the ADIPOQ gene (rs1501299) among patients with DM2 and MS was GT polymorphism G276T. The most frequent genotypes of the ADIPOR1 gene were AC (rs2275737) - 53.3\% and CT (rs2275738) $50.0 \%$. The most frequent combinations between ADIPOR1 and ADIPOQ genes were AC (rs2275737)/CT (rs2275738) and GT (rs1501299) - 28.6\%, as well as AC (rs2275737)/CT (rs2275738) and GG (rs1501299) - 19.7\%. Thus, in patients with MS and DM2 in the Republic of Crimea, associations of polymorphism of the genes of the AN receptors with the development of this pathology have been established. It is known that polyphenols of red grape varieties are epigenetic modifiers. Therefore, these products can be included in individual nutrition programs in population groups genetically predisposed to MS and DM2.
\end{abstract}

\section{Introduction}

The problem of overweight and obesity currently stays critical. Epidemiological data indicate an increase in the number of obese people by $5-7 \%$ per year [1]. Visceral adipose tissue (VAT) in obesity produces a greater amount of reactive oxygen species (ROS), and various inflammatory cytokines, leading to the development of systemic inflammatory response

\footnotetext{
* Corresponding author: julianashramko@rambler.ru
} 
syndrome (SIRS), insulin resistance (IR), metabolic syndrome (MS) and type 2 diabetes mellitus (DM2). Cytokines synthetized by VAT modify insulin production, alter the transduction of the insulin signal in peripheral cells, the activity of insulin receptors, and the intensity of lipogenesis; adiponectin (AN) is of one the most important among the named cytokines. It is involved in the regulation of various metabolic processes, including glucose metabolism and fats' breakdown. ADIPOR1 and ADIPOR2 have been identified as two structurally related adiponectin receptors. The level of AN in the blood serum negatively correlates with numerous risk factors for cardiovascular diseases (CVD); a lower level of AN is considered an independent risk factor for MS [2].

Despite the understanding of the significant role of hereditary factors in the formation of MS and DM2, the genetic component responsible for their development has not been fully established yet. Numerous meta-analyses of population data do not provide an unambiguous answer to the question of genetic defects in both MS and DM2.

Thus, the review [3] indicates that the polymorphism $\mathrm{G}$ of the ADIPOQ $+45 \mathrm{~T}>\mathrm{G}$ allele is associated with a significantly increased risk of DM2 in the Asian population. The rs266729 G-allele in the AN gene increases the risk of DM2 according to the study [4]. A decrease in ADIPOQ expression and a simultaneous increase in leptin expression were found by analyzing adipocytes' transcriptome obtained from VAT in patients with MS [5].

In ADIPOQ, two polymorphisms - T45G (rs2241766) in the second exon and G276T (rs1501299) in the second intron - were investigated in connection with the risk of hypertension and AN circulating changes [6]. The risk of MS, DM-2, hyperglycemia and dyslipidemia in Kyrgyz women is associated with the carriership of the T allele in ADIPOQ gene's polymorphic marker G276T [7]. In the Russian population, separate candidate genes were determined as increasing both the risk of obesity (allele T of the rs2241766 marker of the ADIPOQ gene) and the risk of developing IR (the rs 16928751 marker of the ADIPOR2 gene) [8]. The association of ADIPOQ gene's rs1501299 single nucleotide polymorphism with body weight, IR, serum adipokines' levels and MS was studied by deLuis DA et al. [9]. It was found that insulin level was higher in carrierships of the T-allele than in carrierships without the T-allele. In addition, the total level of adiponectin was found lower in carrierships of the T-allele than in carrierships without the T-allele. Logistic regressive analysis demonstrated that subjects with the T allele were associated with an increased risk of MS. Besides that, cases of DM2, according to [10], were more frequent among homozygotes of ADIPOR1 CC rs2275737.

Obviously, the ambiguity of the results is associated with the complex nature of MS and DM2 as multifactorial diseases.

Treatment and prevention of both MS and DM2 with synthetic medications bring serious difficulties due to their negative effects, high cost, inefficiency in some cases and insufficient availability for many people around the world. In this regard, there is a stable interest in socalled "functional food" - ordinary or everyday food that has positive effects on the target function (functions) beyond the nutritional value / basic nutrition, potentially reduces the risk of diseases and has permitted and scientifically based health requirements. [10]. Multitude of researchers state the correlation between both medicinal plants and functional foods' antioxidant activity with their content of polyphenols $[11,12]$. Phenolic compounds attract abundant attention by reason of their application is associated with the prevention of certain chronic and degenerative diseases, such as CVD and DM2, being main causes of death and disability worldwide [13]. Originally defined as antioxidants, polyphenols are designated in recent studies as epigenetic modifiers [14]. There were given evidences that some flavonoids both have an inhibitory effect on adipogenesis during the differentiation of preadipocytes into adipocytes, accompanied by a drop in PPAR $\gamma$ regulation $[15,16]$, and decline the level of serum AN [17]. Grapes are one of the most important sources of polyphenols among fruits [18]. Our studies have determined the positive effect of grape polyphenols in the correction 
of experimental MS [19], as well as confirmed their role as functional foods in the rehabilitation of patients with CVD [20]. Hence, there is a necessity for further studies of MS and DM2 polymorphic genetic markers, with connection of their interactions, relationship between hereditary predisposition and environmental factors, and methods of non-drug cure by polyphenolic functional foods.

\section{Materials and Methods}

We have studied the polymorphisms of the ADIPOQ - G276T (rs1501299) gene and the polymorphism of the ADIPOR1 gene - A/T (rs2275737) and C/T (rs2275738). The selection of these variants of polymorphism was conditioned by the data of metaanalyses $[6,10,21]$ according to the frequency of associations with the risk of MS and DM2. One hundred patients with confirmed diagnosis of DM2 were included in the study. All patients signed an informed consent to the studies in the prescribed form. Genomic DNA was isolated from the whole blood of patients using the phenol-chloroform method. Real-Time Polymerase Chain Reaction (PCR) on a Biorad CFX96 thermal cycler determined gene polymorphisms. In the reaction mixture were used $70 \mathrm{mM}$ of Tris $\mathrm{HCl}, \mathrm{pH} 8.816 .6 \mathrm{mM}$ of ammonium sulfate 250 $\mathrm{nM}$ fluorescent probes, 1.5 units of Taq DNA polymerases, oligonucleotide primers of Synthol and 50-100 ng of genomic DNA. Amplification was processed at $950 \mathrm{C}$ in $2 \mathrm{~min}$, primer annealing $+45 \mathrm{~T} / \mathrm{G}(\mathrm{rs} 2241766)$ at $650 \mathrm{C},+276 \mathrm{G} / \mathrm{T}(\mathrm{rs} 1501299),+219 \mathrm{~A} / \mathrm{T}$ (rs11061971) and +795 G/A (rs16928751) at 58 0C, $-102 \mathrm{~T} / \mathrm{G}(\mathrm{rs} 2275737)$ and $-106 \mathrm{~T} / \mathrm{S}$ (rs2275738) at $590 \mathrm{C}$, during 40 cycles. The fluorescent dyes used in the probes were FAM (carboxyfluorescein) and HEX (hexachlorofluorescein); the fluorescence quencher was BHQ-1.

The study was performed in the Center for Collective Use of Scientific Equipment "Molecular Biology" of S. I. Georgievsky Medical Academy (structural division) of the V. I. Vernadsky CFU. Investigations were carried out following the rules of the Declaration of Helsinki of 1975, revised in 2013 and approved by the V.I. Vernadsky Crimean Federal University Ethics Committee (Protocol № 8 from 17 Jan. 2018). Data obtained in this study were analyzed using the Statistica 8.0 software package. The frequencies of allelic variants were carried out by the Pearson method with the calculation of the value $\chi^{2}$.

\section{Results}

In our study was stated that the most common genotype of the ADIPOQ gene (rs1501299) among patients with MS was GT polymorphism of G276T with the frequency of $60.8 \%$ (Table 1). The genotype of GG of G276T polymorphism was found in $31.6 \%$ of patients with MS. TT genotype of G276T polymorphism was the most seldom - 7.6\%. The most frequent allele among patients with MS was G allele of G276T polymorphism with the frequency of $62.1 \%$.

Table 1. Frequency of the G276T polymorphism of the ADIPOQ gene

(rs1501299) alleles and genotypes' distribution in patients with MS.

\begin{tabular}{|c|c|c|}
\hline Alleles and genotypes & Absolute values & Relative values \\
\hline \multicolumn{3}{|c|}{ Alleles } \\
\hline G & 98 & 62.1 \\
\hline T & 60 & 37.9 \\
\hline \multicolumn{3}{|c|}{} \\
\hline GG & Genotypes \\
\hline GT & 25 & 31.6 \\
\hline TT & 48 & 60.8 \\
\hline
\end{tabular}


Analyzing the frequency of ADIPOR1 gene genotypes' occurrence in patients with MS, the most frequent genotypes AC (rs2275737) - 53.3\% and CT (rs2275738) - 50.0\% (Table 2) were found. The rarest were the genotype of AA polymorphism (rs2275737) -in 19.5\% of patients with MS, and TT (rs2275738) - in 20.2\%. The most frequent alleles were found C (rs2275737) and C (rs2275738) - in 53.8\% and 54.7\%, respectively.

Table 2. Frequency of ADIPOR1 gene polymorphism rs2275737 and rs 2275738 alleles and genotypes' distribution the in patients with MS.

\begin{tabular}{|l|l|l|c|c|c|}
\hline rs2275737 & $\begin{array}{l}\text { Absolute } \\
\text { values }\end{array}$ & Relative values & rs2275738 & $\begin{array}{l}\text { Absolute } \\
\text { values }\end{array}$ & Relative values \\
\hline \multicolumn{5}{|c|}{ Alleles } \\
\hline A & 85 & 46.2 & C & 103 & 54.7 \\
\hline C & 99 & 53.8 & T & 85 & 45.2 \\
\hline \multicolumn{5}{|c|}{ Genotypes } \\
\hline AA & 18 & 19.5 & CC & 28 & 29.8 \\
\hline AC & 49 & 53.3 & CT & 47 & 50.0 \\
\hline CC & 25 & 27.2 & TT & 19 & 20.2 \\
\hline
\end{tabular}

The most frequent combinations of ADIPOR1 gene variants were found AC (rs2275737) / CT (rs2275738) - 50.5\% of patients with MS. The next most frequent combinations were CC (rs2275737) / CC (rs2275738) - 26.4\% and AA (rs2275737) / TT (rs2275738) - 18.7 \%. The seldom combinations in our study were found AC (rs2275737) / CC (rs2275738) - 3.3\% and CC (rs2275737) / TT (rs2275738) - $1.1 \%$.

When considering the frequency of occurrence of combinations between allelic variants of genes ADIPOQ and ADIPOR1 was shown the following (tab. 3).

Table 3. Frequency of ADIPOR1 gene polymorphism (rs2275737) and (rs2275738) and the ADIPOQ gene polymorphism (rs1501299) genotypes combinations' distribution in patients with MS.

\begin{tabular}{|c|c|c|c|c|c|c|c|c|}
\hline \multirow{3}{*}{ ADIPOR1 (rs2275737) / (rs2275738) } & \multirow{2}{*}{\multicolumn{2}{|c|}{ Totally }} & \multicolumn{6}{|c|}{ ADIPOQ (rs1501299) } \\
\hline & & & \multicolumn{2}{|c|}{ GT } & \multicolumn{2}{|c|}{ GG } & \multicolumn{2}{|c|}{ TT } \\
\hline & $\mathrm{n}$ & $\%$ & $\mathrm{n}$ & $\%$ & $\mathrm{n}$ & $\%$ & $\mathrm{n}$ & $\%$ \\
\hline $\mathrm{AC}(\mathrm{rs} 2275737) / \mathrm{CT}(\mathrm{rs} 2275738)$ & 46 & 50.5 & 26 & 28.6 & 18 & 19.7 & 2 & 2.2 \\
\hline $\mathrm{CC}(\mathrm{rs} 2275737) / \mathrm{CC}(\mathrm{rs} 2275738)$ & 24 & 26.4 & 13 & 14.3 & 10 & 11.0 & 1 & 1.1 \\
\hline AA (rs2275737) / TT (rs2275738) & 17 & 18.7 & 7 & 7.7 & 9 & 9.9 & 1 & 1.1 \\
\hline $\mathrm{AC}(\mathrm{rs} 2275737) / \mathrm{CC}(\mathrm{rs} 2275738)$ & 3 & 3.3 & 1 & 1.1 & 1 & 1.1 & 1 & 1.1 \\
\hline CC (rs2275737) / CT (rs2275738) & 1 & 1.1 & 0 & 0 & 1 & 1.1 & 0 & 0 \\
\hline Totally & 91 & 100 & 47 & 51.7 & 39 & 42.8 & 5 & 5.5 \\
\hline
\end{tabular}

In MS patients, the most frequent combinations between ADIPOR1 and ADIPOQ genes were AC (rs2275737) / CT (rs2275738) and GT (rs1501299) - 28.6\%, as well as AC (rs2275737)/CT (rs2275738) and GG (rs1501299) - 19.7\%.

\section{Conclusion}

Hence, in the Crimean population, a high degree of association of genes' polymorphisms with the development of DM2 and MS was confirmed, that consistent with the data of the existed meta-analyses. This is the basis for MS and DM2 risk group formation in the Crimean population, as well as for the individualization of nutritional correction programs' applying polyphenolic grape processing products. 


\section{References}

1. NCD Risk Factor Collaboration (NCD-RisC). Trends in adult body-mass index in 200 countries from 1975 to 2014: A pooled analysis of 1698 population-based measurement studies with 19.2 million participants. Lancet, 387, 1377-1396 (2016)

2. P. Li, R. Jiang, L. Li, C. Liu, F. Yang, Y. Qiu, Eur. J. Clin. Nutr., 69(1), 62-7 (2015)

3. Y. Fan, K. Wang, S. Xu, G. Chen, H. Di, M. Cao, C. Liu, Int.J.Mol.Sci., 16 (1), 704-23 (2014)

4. Sun P, Liu L, Chen J, Chen Y, Shi L, Imam MU, Chen Y, Pei X, Xu Y, Guo Y, Ping Z, Fu X.. Medicine, 96 (47), e8745 (2017)

5. S. Gormez, A. Demirkan, F.Atalar, B.Caynak, R.Erdim, V. Sozer, et al. Intern.Med., 50(8), 805-910 (2011)

6. J. Wu, G. Xu, W. Cai, et.al. ONCOTARGET, 8(9), 14636-14645 (2017)

7. Z.T. Isakova, E.T.Talaibekova, O.S. Lunegova, D.A. Asambarva, A.S. Kerimkulova, A.A. Aldashev, Probl. Endokrinol. (Mosk), 61(1), 46-50 (2015)

8. K. A. Vakhromeeva, Polymorphic genetic markers of type 2 diabetes mellitus and their associations with clinical and metabolic parameters in the Russian population (Cand. med. sci. diss. Abstr. Tyumen', 23, 2015)

9. D.A. de Luis, O. Izaola, B. de la Fuente, D. Primo, H. Fernandez Ovalle, E. Romero, Ann. Nutr. Metab., 69(3-4), 226-231 (2016)

10. H. Wang, H. Zhang, Y. Jia, Z. Zhang, R. Craig, X. Wang, S.C. Elbein. Diabetes. 53(8), 2132-2136 (2004)

11. V. Tufarelli, V. Laudadio, J. Exp. Biol. Agric. Sci.,6, 273-278 (2016)

12. D. P. Xu, Y. Li, X. Meng, T.Zhou, Y.Zhou, J.Zheng, J. J.Zhang, and H. B. Li, Int. J. Mol. Sci., 18(1), 96 (2017)

13. Ya. I. Yashin, A. N.Vedenin, A. Ya. Yashin, Sorbtsionnye i Khromatograficheskie Protsessy, 3(17), 496-505 (2017)

14. C.Santos-Buelga, A. M. González-Paramás, T.Oludemi, B. Ayuda-Durán, and S. González-Manzano, Adv. Food Nutr. Res., 90, 183-257 (2019)

15. M.L. Ricketts, B.S. Ferguson, Curr. Pharm. Des., 24(2), 158-170 (2018)

16. S.J. Lee, Y. Jia, Curr. Opin. Lipidol, 26(1), 3-9 (2015)

17. D. Różańska, B.Regulska-Ilow, Adv.Clin.Exp.Med., 27(1),135-142 (2018)

18. A. Antoniolli, A. R. Fontana, P. Piccoli, R.Bottini, Food Chem., 178, 172-178 (2015)

19. A. V. Kubyshkin, Yu. I. Shramko, E. Yu. Zyablitskaya, V. I. Petrenko, N. A. Ivaschenko, C. O. Tarimov, I. V. Chernousova, Yu. A. Ogay, Medical News North Caucasus, 15(4), 563-567(2020)

20. I. V. Chernousova, G. P. Zaytsev, V. E. Mosolkova et al., Magarach Vinogradstvo i Vinodelie, 49, 258-260 (2020)

21. L. Yang, W. Ling, Y. Qiu, et al. Nutr. Metab., 17, 78 (2020) 